\title{
Importance of radiotherapy to the primary in metastatic hormone sensitive prostate cancer
}

\author{
Elisha T. Fredman ${ }^{1} \cdot$ Gordon Guo $\mathbb{1}^{1} \cdot$ Daniel E. Spratt $\mathbb{D}^{2}$
}

Received: 15 February 2021 / Revised: 11 March 2021 / Accepted: 11 March 2021 / Published online: 8 April 2021

(C) The Author(s), under exclusive licence to Springer Nature Limited 2021

In the evolving paradigm of metastatic prostate cancer treatment, an unanswered question remains regarding the role of treating the primary tumor and its impact on the natural history of the disease. In hindsight, there was likely early data to support the role of radiotherapy (RT) to the primary in metastatic prostate cancer. The 2011 NCIC/ MRC PRO7 trial, asked the question of whether men with aggressive locally advanced prostate cancer benefited from the addition of RT to androgen deprivation therapy (ADT) [1]. Although M1 patients were technically excluded, bone scintigraphy was not mandated. Patients on this trial had very aggressive disease (83\% had clinical T3 and $4 \%$ had T4 disease, $>50 \%$ had a PSA $>20 \mathrm{ng} / \mathrm{mL}$ and $25 \%$ had a PSA of $>50 \mathrm{ng} / \mathrm{mL}$ ) and in retrospect were at high risk of harboring detectable metastatic disease with either conventional or current advanced molecular imaging. Despite the liberal inclusion criteria, a clear and statistically significant survival benefit was still seen with the addition of RT to the primary.

Multiple population registry studies also demonstrated a potential benefit of RT to the primary in men with M1 disease by conventional imaging. For example, in a study using the National Cancer Database ( $n=6382$ patients) with mHSPC showed that in the subset $(n=538)$ that received RT to the primary, there was a significant overall survival benefit (HR 0.62; 95\% CI 0.55-71, $p<0.001$ ) [2]. Given the well-known selection biases in such registries, this work, while not conclusive, was hypothesis generating.

The first randomized trial to investigate the potential benefit of adding RT to the primary in de novo mHSPC to

Daniel E. Spratt

sprattda@umich.edu

1 Department of Radiation Oncology, University Hospitals, Seidman Cancer Center, Cleveland, OH, USA

2 Department of Radiation Oncology, University of Michigan, Ann Arbor, MI, USA standard of care systemic therapy was the HORRAD trial [3], which randomized 432 men to $\mathrm{ADT} \pm \mathrm{RT}$ to the primary. Eligibility requirements included evidence of bone metastases and a PSA $>20 \mathrm{ng} / \mathrm{mL}$. After a median followup of 47 months there was no improvement in OS from the addition of RT to the primary (HR $0.90 ; 95 \% \mathrm{CI}$, $0.70-1.14)$. On this trial, the median PSA was 142 and $67 \%$ of patients had $>5$ bone metastases. In an unplanned subset analysis, the authors demonstrated a potential interaction between volume of metastatic disease and benefit of treating the primary. Patients with low volume disease had signal for potential OS benefit (HR 0.68; 95\% CI, 0.42-1.10).

This promising result from the HORRAD study was further brought to light in the much larger Systemic Therapy in Advancing and Metastatic Prostate cancer (STAMPEDE) trial, Arm H, which tested the hypothesis that the addition of RT to the prostate for mHSPC patients would improve OS [4]. In 2000 patients, there was no significant difference in 3-year OS by treatment arm in the total study population (HR $0.92,95 \%$ CI $0.80-1.06 ; p=0.266$ ). There was, however, a failure-free survival benefit from the addition of RT to the primary (HR $0.76,95 \%$ CI $0.68-0.84$; $p<0.0001)$. Importantly, there were no significant difference in grade $\geq 3$ toxicity with the addition of RT (HR 1.01; 95\% CI, 0.87-1.16; $P=0.94)$. On subset analysis, among the $40 \%$ of patients with a low metastatic burden, there was a significant 3-year OS benefit with the addition of RT to the prostate (HR 0.68, 95\% CI 0.52-0.90; $p=0.007$ ).

Based on the totality of the evidence, guidelines now support RT to the primary in low metastatic burden mHSPC as an accepted standard of care [5]. As with any new treatment option, we are left with many unanswered questions,

- Would the demonstrated benefits persist over time (HORRAD and STAMPEDE had relatively short follow-up)?

- Will further systemic therapy intensification further improve OS with treatment of the primary? 
- What is the optimal dose of prostate-directed RT?

- What is the role of metastases-directed RT?

The current study by Morgan et al. sheds new light on some of these questions [6]. This group reports one of the largest single institution series of patients with de novo mHSPC, with relatively long-term follow-up. Their cohort included 410 patients, of which 128 received RT to the primary, and had a median follow-up of 61 months, with some patients followed $>10$ years. As expected in a retrospective analysis, there were baseline characteristic differences between those who did and did not receive RT consistent with selection bias. After accounting for many known confounders on multivariable analysis, they demonstrated an OS advantage in the cohort receiving RT to the primary (median OS 47.7 vs 26.3 months, HR 0.69, 95\% CI $0.50-0.94, p=0.02)$. This difference was even more pronounced among patients surviving at least 1 year (median OS 52.2 vs 39.8 months, HR 0.73 , 95\% CI $0.54-0.98, p=0.04)$. Of note, the benefits of RT to the primary persisted even out to 5- and 7-years post-treatment.

The authors also report an association between higher biologically equivalent dose of radiation and improved OS. Although interesting, caution should be exercised in interpreting this data due to the presence of potentially confounding factors, many appropriately identified by the authors. Baseline patient characteristics stratified by dose of RT are not provided, and may highlight the selection bias. Dose escalation in localized disease has repeated been shown to not improve OS, and it remains unclear how dose escalation in mHSPC could impact OS. However, a plausible explanation for improved OS in mHSPC may be that higher RT dose was simply a surrogate of better performance status, lower volume disease, and/or favorable response to systemic therapy. It should be noted as well that the doses in this study were relatively low dose and did not dose escalate much beyond the doses used in STAMPEDE Arm H. Given the favorable toxicity data in STAMPEDE
Arm $\mathrm{H}$, we still lack evidence to further dose escalate beyond doses used on STAMPEDE in the mHSPC setting ( $55 \mathrm{~Gy} / 20$ fractions or $36 \mathrm{~Gy} / 6$ fractions).

We congratulate Dr. Morgan et al. and are encouraged by the consistent data showing an OS benefit from RT to the primary in the setting of mHSPC.

\section{Compliance with ethical standards}

Conflict of interest The authors declare no competing interests. Nonrelevant COI: DES- Personal Fees from Janssen, AstraZeneca, Boston Scientific, and Blue Earth. Funding from Janssen.

Publisher's note Springer Nature remains neutral with regard to jurisdictional claims in published maps and institutional affiliations.

\section{References}

1. Warde P, Mason M, Ding K, Kirkbride P, Brundage M, Cowan R, et al. Combined androgen deprivation therapy and radiation therapy for locally advanced prostate cancer: a randomised, phase 3 trial. Lancet. 2011;378:2104-11.

2. Rusthoven CG, Jones BL, Flaig TW, David Crawford E, Koshy M, Sher DJ, et al. Improved survival with prostate radiation in addition to androgen deprivation therapy for men with newly diagnosed metastatic prostate cancer. J Clin Oncol. 2016;34:2835-42.

3. Boevé LM, Hulshof MC, Vis AN, Zwinderman AH, Twisk JWR, Witjes WPJ, et al. Effect on survival of androgen deprivation therapy alone compared to androgen deprivation therapy combined with concurrent radiation therapy to the prostate in patients with primary bone metastatic prostate cancer in a prospective randomised clinical trial: data from the HORRAD trial. Eur Urol. 2019;75:410-8.

4. Parker CC, James ND, Brawley CD, Clarke NW, Hoyle AP, Ali A, et al. Radiotherapy to the primary tumour for newly diagnosed, metastatic prostate cancer (STAMPEDE): a randomised controlled phase 3 trial. Lancet. 2018;392:2353-66.

5. NCCN Guidelines, Version 1.2021: https://www.nccn.org/ professionals/physician_gls/pdf/prostate.pdf.

6. Morgans SC, Holmes OE, Craig J, et al. Long-Term Outcomes of Prostate Radiotherapy for Newly-Diagnosed Metastatic HormoneSensitive Prostate Cancer. Prostate Cancer Prostatic Dis. 2021. Online ahead of print. 